\title{
Laguerre Expansions for Successive Generations of a Renewal Process
}

\author{
George H. Weiss
}

(August 17, 1962)

\begin{abstract}
It is shown that the coefficients in the Laguerre expansions for successive generations in a renewal process are related by an algebraic convolution. Thus the calculations are easily mechanized for computation.
\end{abstract}

In a paper published in 1940 , Brown $[1]^{1}$ studied the solution of the renewal equation under the assumption that the functions appearing in it could be represented by a Pearson type III function. The natural generalization of this type of solution is to assume that the functions involved in the renewal equation can be expanded in terms of a series of generalized Laguerre functions. Recently Zelen and Dannemiller [2] have presented a summary of results concerning the expansion of reliability functions in terms of generalized Laguerre functions, but did not discuss these expansions in the context of their application to renewal equations. In view of the increasing number of applications of renewal theory and the theory of semi-Markov processes [3-6] to stochastic models in reliability, biology, and the theory of traffic it is of some interest to return once more to the subject of approximate solutions to the renewal equation. In this note we shall outline some of the results relating solutions of the renewal equation and expansion into series of Laguerre functions, without going too deeply into matters of uniqueness and convergence of the resulting series.

We shall consider the simple renewal equation:

$$
\varphi(t)=f(t)+\int_{0}^{t} \varphi(\tau) g(t-\tau) d \tau
$$

and assume in what follows the existence of a unique solution to this equation. For most applications the functions $f(t)$ and $g(t)$ are assumed to be nonnegative over the interval $0 \leq t<\infty$ and the definite integrals

$$
\int_{0}^{\infty} f(t) d t, \int_{0}^{\infty} g(t) d t
$$

are assumed to be bounded. A formal solution to eq (1) can be given in terms of the Laplace transforms of $f(t)$ and $g(t)$, and provided that $f(t)$ and $g(t)$ are measurable, nonnegative, and bounded in every interval, it can be shown that a unique nonnegative

\footnotetext{
1 Figures in brackets indicate the literature references at the end of this paper.
}

solution of the renewal equation indeed exists. Furthermore the asymptotic properties of $\varphi(t)$ can be obtained from the properties of the individual Laplace transforms [7].

If we define the following Laplace transforms

$$
\begin{aligned}
& \varphi^{*}(s)=\int_{0}^{\infty} e^{-s t} \varphi(t) d t \\
& f^{*}(s)=\int_{0}^{\infty} e^{-s t} f(t) d t \\
& g^{*}(s)=\int_{0}^{\infty} e^{-s t} g(t) d t
\end{aligned}
$$

then the transformed solution to eq (1) can be written

$$
\varphi^{*}(s)=\frac{f^{*}(s)}{1-g^{*}(s)} .
$$

This solution has been used by Feller [7] to discuss in a rigorous manner an expansion first proposed by Lotka. The solution in question can be written

$$
\varphi(t)=\sum A_{k} e^{s} k^{t}
$$

where it is assumed that there is a denumerable sequence of distinct roots, $s_{0}, s_{1}, s_{2}, \ldots$ to the equation

$$
g^{*}(s)=1 .
$$

Feller proves the following theorem:

In order that the solution to eq (1) be expressible in the form of eq (3), where the series converges absolutely for $\mathrm{t} \geq 0$ it is necessary and sufficient that the Laplace transform $\phi^{*}(s)$ admit the expansion

$$
\varphi^{*}(s)=\frac{f^{*}(s)}{1-g^{*}(s)}=\sum_{k} \frac{A_{k}}{s-s_{k}}
$$

and that $\Sigma\left|\mathrm{A}_{\mathrm{k}}\right|$ converges absolutely. The coefficients $\mathrm{A}_{\mathrm{k}}$ are determined by

$$
A_{k}=-f^{* \prime}\left(s_{k}\right) / g^{*}\left(s_{k}\right) .
$$


It is easy to generalize this theorem to cover the possibility of having multiple roots. One can see that the conversion of the renewal equation into an algebraic equation in the transform domain leads to a simple representation of the solution. We shall see that this simplification is retained when $f(t)$ and $g(t)$ are expanded in terms of generalized Laguerre functions. Hadwiger [8] has shown that if $f(t)$ and $g(t)$ are represented by Taylor series in a finite interval and vanish outside of the interval then the renewal function can also be expanded as a Taylor series and the coefficients obey a discrete convolution relation. This result is very similar to the results to be presented here.

To begin our exposition, let us recast eq (1) into a slightly different form by defining the successive generation functions $\left\{\varphi_{k}(t)\right\}$ by

$$
\begin{aligned}
\varphi_{0}(t) & =f(t) \\
\varphi_{n+1}(t) & =\int_{0}^{t} \varphi_{n}(\tau) g(t-\tau) d \tau, n=0,1,2, \ldots
\end{aligned}
$$

The solution, $\varphi(t)$, is then,

$$
\varphi(t)=\sum_{n=0}^{\infty} \varphi_{n}(t)
$$

Clearly the $\varphi_{n}(t)$ are also the successive terms in a solution to eq (1) by means of successive approximations. If we define the Laplace transforms

$$
\varphi_{n}^{*}(s)=L\left\{\varphi_{n}(t)\right\}
$$

then the Laplace transform of eq (7) is

or

$$
\varphi_{0}^{*}(s)=f^{*}(s), \varphi_{n+1}^{*}(s)=\varphi_{n}^{*}(s) g^{*}(s)
$$

$$
\varphi_{n}^{*}(s)=f^{*}(s)\left[g^{*}(s)\right]^{n} .
$$

We shall essentially use the forms of the renewal equation given in this paragraph.

The generalized Laguerre functions $L_{n}^{m}(x)$ will be defined by

$$
\begin{aligned}
L_{n}^{m}(t) & =\frac{1}{n !} e^{t} t^{-m} \frac{d^{n}}{d t^{n}}\left(e^{-t} t^{n+m}\right) \\
& =\sum_{j=0}^{n}\left(\begin{array}{c}
n+m \\
n-j
\end{array}\right) \frac{(-t)^{j}}{j !}
\end{aligned}
$$

for $n$ an integer. These functions have the normalization property

$$
\int_{0}^{\infty} e^{-t} t^{m} L_{r}^{m}(t) L_{s}^{m}(t) d t=\frac{\Gamma(m+r 1+)}{r !} \delta_{r s}
$$

and their Laplace transforms take the form

$$
\int_{0}^{\infty} e^{-u t} t^{m} L_{n}^{m}(t) d t=\frac{\Gamma(m+n+1)}{n !} \frac{(u-1)^{n}}{u^{m+n+1}} .
$$

We shall assume that the functions $f(t)$ and $g(t)$ can be expanded into series of the generic form

$$
p(t)=\frac{e^{-\lambda t}}{\Gamma(m+1)} \lambda^{m+1} t^{m} \sum_{n=0}^{\infty} a_{n} L_{n}^{m}(\lambda t)
$$

where $\lambda$ is a parameter with dimensions of $t^{-1}$. It is put in because in most applications the functions $p(t)$ are densities. The best results on the pointwise convergence of this series are contained in a paper by Uspensky [9]. He proved that the following conditions are sufficient for pointwise convergence (except at discontinuities, where the sum converges to $\frac{1}{2}(p(t+)+p(t-))$ :

1. $\int_{r}^{\infty} t^{m} e^{-t} p^{2}(t) d t$ exists for a certain $r$.

2. For $-1<m \leq-\frac{1}{2} \int_{0}^{\beta} t^{m} e^{-t} p^{2}(t) d t$ exists for a certain $\beta$ while for $m>-\frac{1}{2}$ only

$$
\int_{0}^{\beta} t^{\frac{m}{2}-\frac{1}{4}}|p(t)| d t
$$

exists.

3. The function $p(t)$ is of bounded variation and absolutely integrable in any finite interval. In the cases of present interest most functions $p(t)$ will satisfy these three criteria. In particular if the function $f(t)$ and $g(t)$ are bounded over $(0, \infty)$ then conditions 1 and 2 will always be satisfied. Specific applications of this expansion have been made in references [11] and [12].

If the expansion of eq (15) is assumed to exist then the coefficients $a_{n}$ are given by

$$
\begin{aligned}
a_{n} & =\frac{1}{\left(\begin{array}{c}
m+n \\
n
\end{array}\right)} \int_{0}^{\infty} L_{n}^{m}(\lambda t) p(t) d t \\
& =\frac{1}{\left(\begin{array}{c}
m+n \\
n
\end{array}\right)} \sum_{j=0}^{n}\left(\begin{array}{c}
m+n \\
n-j
\end{array}\right) \frac{(-1)^{j}}{j !} \lambda^{j} \mu_{j}
\end{aligned}
$$

where the $\mu_{j}$ are the moments

$$
u_{j}=\int_{0}^{\infty} t^{j} p(t) d t
$$

We will assume that all of the functions $p(t)$ that are to be treated are such that moments of all orders exist. If $p^{*}(s)$ denotes the Laplace transform of $p(t)$ then an application of eq (14) shows that

$$
p^{*}(s)=\frac{\lambda^{m+1}}{(s+\lambda)^{m+1}} \sum_{n+0}^{\infty} a_{n}\left(\begin{array}{c}
m+n \\
n
\end{array}\right) \frac{s^{n}}{(s+\lambda)^{n}} .
$$

Thus we see that in the $s$ domain the expansion of $p^{*}(s)$ is essentially a power series development in the variable $s /(s+\lambda)$.

Now let us assume that $f(t)$ and $g(t)$ have the expansions 


$$
\begin{aligned}
& f(t)=\frac{e^{-\lambda t} \lambda^{m+1} t^{m}}{\Gamma(m+1)} \sum_{n=0}^{\infty} f_{n} L_{n}^{m}(\lambda t) \\
& g(t)=\frac{e^{-\lambda t} \lambda^{r+1} t^{r}}{\Gamma(r+1)} \sum_{n=0}^{\infty} g_{n} L_{n}^{r}(\lambda t)
\end{aligned}
$$

and calculate the coefficients in the expansion of $\varphi_{j}(t)$. The simplest procedure is to use Laplace transforms. In this way we find

$$
\varphi_{k}(s)=\frac{\lambda^{m+1+k(r+1)}}{(s+\lambda)^{m+1+k(r+1)}} \sum_{j=0}^{\infty} c_{j}^{(k)}\left(\frac{s}{s+\lambda}\right)^{j}
$$

where the $c_{r}^{(k)}$ are constants which satisfy the recurrence relations

$$
\begin{aligned}
c_{j}^{(0)} & =\left(\begin{array}{c}
m+j \\
j
\end{array}\right) f_{j} \\
c_{i}^{(k+1)} & =\sum_{l=0}^{j} c_{j-l}^{(k)}\left(\begin{array}{c}
r+j \\
j
\end{array}\right) g_{i} .
\end{aligned}
$$

That is to say, the c's may be found by successive convolutions with the sequence $\left\{\left(\begin{array}{c}r+j \\ j\end{array}\right) g_{j}\right\}$. Thus the convolution integrals are converted into more convenient algebraic convolutions.

If the inverse transform of eq (11) is taken, the expansion of $\varphi_{k}(t)$ in the time domain becomes

$$
\begin{aligned}
\varphi_{k}(t)=e^{-\lambda t} \sum_{j=0}^{\infty} c_{j}^{(k)} \frac{1}{\left(\begin{array}{c}
m+k(r+1)+j \\
m+k(r+1)
\end{array}\right)} \\
\frac{\lambda^{m+k(r+1)+1} t^{m+k(r+1)}}{(m+k(r+1)) !} L_{j}^{m+k(r+1)}(\lambda t) .
\end{aligned}
$$

The most useful case in practice is that for which $m=r=0$, i.e., an expansion in terms of Laguerre functions of order zero. For this special case one has

$$
\varphi_{k}(t)=e^{-\lambda t} \sum_{j=0}^{\infty} c_{j}^{(k)} \frac{1}{\left(\begin{array}{c}
k+j \\
k
\end{array}\right)} \frac{\lambda^{k+1} t^{k}}{k !} L_{j}^{k}(\lambda t) .
$$

This expression can be further reduced through the identity

$$
L_{j}^{m}(x)=\sum_{r=0}^{j} L_{r}^{m-1}(x)
$$

or

$$
L_{j}^{m}(x)=\sum_{p=0}^{j}\left(\begin{array}{c}
j-p+m-1 \\
m-1
\end{array}\right) L_{p}(x) \quad m \geq 1 .
$$

On substituting this expression into eq (22) and collecting terms we find

$\varphi_{k}(t)=\frac{\lambda^{k+1} t^{k}}{k !} e^{-\lambda t} \sum_{m=0}^{\infty} L_{m}(\lambda t) \sum_{j=m}^{\infty} \frac{\left(\begin{array}{c}j+k-m-1 \\ m-1\end{array}\right)}{\left(\begin{array}{c}j+k \\ j\end{array}\right)} c_{j}^{(k)}$

$k \geq 1$.
When $g(t)$ is such that only a few of the coefficients $g_{m}$ are nonzero, then the procedure of taking algebraic convolutions is quite simple. For example, if we drop the restriction that $f(t)$ and $g(t)$ be positive, and assume that $f(t)$ and $g(t)$ are given by

$$
\begin{aligned}
& f(t)=a e^{-\lambda t} L_{n}(\lambda t) \\
& g(t)=b e^{-\lambda t} L_{m}(\lambda t)
\end{aligned}
$$

then a direct calculation leads to the result for $\varphi_{k}^{*}(s)$ :

$$
\varphi_{k}^{*}=\frac{\lambda^{k+1} a b^{k} s^{n+k m}}{(s+\lambda)^{n+1+k(m+1)}}
$$

which implies

$$
\begin{aligned}
\varphi_{k}(t)=a b^{k} \frac{\lambda^{k+1}}{k !} t^{k} e^{-\lambda t} \frac{1}{\left(\begin{array}{c}
n+k m+k \\
k
\end{array}\right)} L_{n+k m}^{k}(\lambda t) \\
=a b^{k} \frac{\lambda^{k+1}}{k !} t^{k} e^{-\lambda t} \frac{1}{\left(\begin{array}{c}
n+k(m+1) \\
k
\end{array}\right)} \sum_{j=0}^{n+k m} \\
\left(\begin{array}{c}
k+n+k m-j-1 \\
k-1
\end{array}\right) L_{j}(\lambda t) .
\end{aligned}
$$

The simplest way to find $\varphi(t)$ is to sum over $k$ in the transform. This leads to a result analogous to that of Lotka in eq (3). If $f(t)$ and $g(t)$ are given by

$$
f(t)=g(t)=e^{-\lambda t}\left(1+a L_{n}(\lambda t)\right)
$$

then it is easily verified by induction that

$$
c_{j}^{(k)}=d_{j}^{(0)}+\left(\begin{array}{c}
k \\
1
\end{array}\right) a d_{j-n}^{(0)}+\left(\begin{array}{l}
k \\
2
\end{array}\right) a^{2} d_{j-2 n}^{(0)}+\ldots
$$

where

$$
d_{j}^{(0)}=c_{j,}^{(0)} j \geq 0, d_{j}^{(0)}=0 \quad, j<0
$$

hence

$$
c_{j}^{(0)}=\delta_{j, 0}+a \delta_{j, n}
$$

we see that $c_{j}^{(k)}=0$ unless $j$ is an integral multiple of $n$. A further calculation starting from eq (31) shows that

$$
c_{r n}^{(k)}=\left(\begin{array}{c}
k+1 \\
r
\end{array}\right) a^{r} \quad r=0,1,2, \ldots k+1 .
$$

With this explicit formula, the infinite sum in eq (23) is reduced to a finite series

$$
\begin{aligned}
\varphi_{k}(t)=\frac{\lambda^{k+1} t^{k}}{k !} e^{-\lambda t} \sum_{m=0}^{(k+n) r+k-1} L_{m}(\lambda t) \sum_{r=\left[\frac{m}{n}\right]}^{k+1} \\
\frac{\left(\begin{array}{c}
m+k-m-1 \\
k-1
\end{array}\right)}{\left(\begin{array}{c}
m+k \\
k
\end{array}\right)}\left(\begin{array}{c}
k+1 \\
r
\end{array}\right) a^{r} .
\end{aligned}
$$


There are several extensions of this method that are possible. It is not too difficult to generalize the solutions here to finite sets of semi-Markov equations (i.e., the matrix analog of eq (1)). It has also been pointed out by Harold Kaplan that discrete renewal equations can also be handled by the same methods provided that we start with Gottlieb polynomials [10] rather than Laguerre functions for the relevant expansions. The obvious advantage of the present method is the fact that one can reduce the integral equation in an exact way to recurrence relations which may then be easily handled by a computer. The method is probably not too good if there are many nonzero coefficients or if only the moments of $f(t)$ and $g(t)$ are known experimentally and the coefficients must be calculated from them. Preliminary experiments show that the results are very sensitive to errors in expressions for higher moments.

\section{References}

[1] A. W. Brown, A note on the use of a Pearson type III function in renewal theory, Ann. Math. Stat. 11, 448 (1940).

[2] M. Zelen and C. Dannemiller, The robustness of life testing procedures derived from the exponential distribution, Technometries 3, 29 (1961).
[3] K. J. Arrow, S. Karlin, and H. Scarf, ed., Studies in the Mathematical Theory of Inventory and Production (Stanford Univ. Press, 1958).

[4] K. J. Arrow, S. Karlin, and H. Scarf ed., Studies in Applied Probability and Management Science (Stanford Univ. Press, 1962).

[5] G. H. Weiss and A. A. Maradudin, Some problems in traffic delay, Operations Research 10, 74 (1962).

[6] G. H. Weiss and M. Zelen, A semi-Markov model for chronic diseases (to be published).

[7] W. Feller, On the integral equation of renewal theory, Ann. Math. Stat. 12, 243 (1941).

[8] H. Hadwiger, Über die Entwicklung Erneuerungsfunktion in eine Potenzreihe, Skand. Aktuarietidskrift 21, 31 (1938).

[9] J. V. Uspensky, On the development of arbitrary functions in series of Hermite's and Laguerre's polynomials, Ann. Math. 28, 593 (1927).

[10] M. J. Gottlieb, Concerning some Polynomials Orthogonal on a Finite or Enumerable set of Points, Am. J. Math. 60, 453 (1938).

[12] M. M. Siddiqui, Some properties of the empirical distribution function of a random process, J. Research NBS 65B (Math. \& Math. Phys.) No. 2, 117 (1961).

[13] J. Gurland, Distribution of quadratic forms and ratios of quadratic forms, Ann. Math. Stat. 24, 416 (1953).

(Paper 66B4-83) 